\title{
Up-Regulation Thioredoxin Inhibits Advanced Glycation End Products-Induced Neurodegeneration
}

\author{
Xiang Ren ${ }^{\mathrm{a}} \quad$ Ni-na Wang ${ }^{\mathrm{a}}$ Hui Qia ${ }^{\mathrm{a}}$ Yuan-yuan Qiu ${ }^{\mathrm{a}} \quad$ Cheng-hong Zhang ${ }^{\mathrm{c}}$ \\ Emily Brown ${ }^{d}$ Hui Kong ${ }^{b}$ Li Kong ${ }^{a}$ \\ aDepartment of Histology and Embryology, College of Basic Medicine, Dalian Medical University, \\ Dalian, bDepartment of Otorhinolaryngology, The Second Hospital of Dalian Medical University, Dalian, \\ cTeaching Laboratory of Morphology, College of Basic Medicine, Dalian Medical University, Dalian, \\ China, dDepartment of Ophthalmology, College of Medicine, University of Florida, Gainesville, USA
}

\section{Key Words}

Thioredoxin $\cdot$ Diabetic retinopathy $•$ Apoptosis $•$ Autophagy

\begin{abstract}
Background/Aims: Diabetic retinopathy (DR) is one of the most serious complications of diabetes and is the leading cause of adult blindness in developed countries. Advanced glycation end products (AGEs) accumulation in diabetes is associated with its complications. Thioredoxin (Trx) is a small molecule $(12 \mathrm{kDa})$ antioxidant protein widely distributed in mammalian tissues, which has important biological functions including anti-apoptosis and transcriptional regulation. In a previous study, we found that Trx plays a key role in retinal neurodegeneration prior to the occurrence of endothelial damage in diabetic mice. In this study, our aim is to determine the effect of Trx on neurodegeneration induced by AGEs in order to identify new therapeutic targets for the clinical treatment and prevention of DR. Methods: In vivo, a high-fat diet and Streptozotocin (STZ) injection were used to generate a mouse model of diabetes. Histology was utilized to examine tissue morphology and measure the outer nuclear layer (ONL) thickness. Electroretinography (ERG) was used to assess retinal function and Western blot was used to examine protein expression. In vitro, three methods of Trx up-regulation were used, including a stable cell line that overexpresses Trx, treatment with Sulforaphane, and shRNA down-regulation Txnip. Cells were treated with AGEs, and level of apoptosis was performed to quantify this by flow cytometry and TUNEL. Quantitative Reverse Transcription PCR (qRT-PCR), Western blotting and immunofluorescence were used to measure gene and protein expression. Transmission electron microscopy (TEM) was used to observe autophagosomes. Results: We found that diabetic mice display decreased retinal function and reduced ONL thickness with AGEs accumulation and a reduction of Trx expression. Up-regulation Trx can prevent the ONL thickness decrease in diabetic mice,

X. Ren, N.-N. Wang and H. Qi contributed equally to this work.

\begin{tabular}{ll}
\hline Li Kong & Department of Histology and Embryology, College of Basic Medicine, Dalian Medical University \\
and Hui Kong & Dalian 116044; Department of Otorhinolaryngology, The Second Hospital of Dalian Medical University, \\
& Dalian 116023 (China); E-Mail kongli@dmu.edu.cn; konghui6905@163.com
\end{tabular}
\end{abstract}




\section{Cellular Physiology Cell Physiol Biochem 2018;50:1673-1686

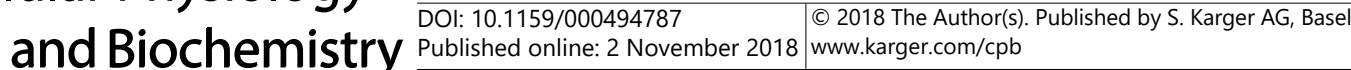 \\ Ren et al.: Trx Inhibited Neurodegeneration}

as observed by H\&E staining. In vitro, up-regulation Trx resulted in decreased intracellular ROS generation, reduced apoptosis by inhibited autophagy. Conclusion: Up-regulating Trx inhibited neurodegeneration induced by AGEs. The underlying mechanism may be related to inhibit Txnip/mTOR pathway-mediated autophagy.

\section{Introduction}

Diabetes mellitus (DM) is one of the most common metabolic diseases in the world, and the morbidity and mortality from the disease are increasing annually. According to the World Health Organization, it is estimated that the total number of people with diabetes will double from 171 to 366 million by 2030[1]. At present, DM can be divided into Type 1 and Type 2. Type 2 diabetes (T2D) is a metabolic disorder characterized by an abnormal regulation of nutrients and nutrient metabolites that develops as a consequence of combined insulin resistance and relative insulin deficiency $[2,3]$. DM can lead a variety of complications, such as nephropathy, angiopathy, retinopathy, and peripheral neuropathy [4].

Diabetic retinopathy (DR) is one of the most serious complications of diabetes and is one of the leading causes of adult blindness in developed countries [5]. With the increasing number of people with diabetes, the number of cases of DR and vision-threatening DR (VTDR), which includes severe non-proliferative DR, proliferative DR (PDR) and diabetic macular edema (DME), has been estimated to rise to 191.0 million and 56.3 million, respectively by 2030[6]. However, recent studies have identified that the loss of function and structure of retina in $\mathrm{db} / \mathrm{db}$ mice, a model of Type 2 diabetes mellitus, occurs prior to detectable alternations of blood vessels [5]. Thus, it is important to understand the mechanisms and different phases of degeneration in order to target diabetic nerve cells in the early stage of DR to prevent and reverse degeneration in cells before cell death occurs. Although various factors contribute to the pathogenesis of diabetic complications in several DM animal models, increased concentrations of advanced glycation end products (AGEs) have been associated with DR[7]. AGEs are the result of a chain of chemical reactions after an initial glycation reaction. The intermediate products are known as Amadori, Schiff base and Maillard products. AGEs have been implicated in the progression of age-related diseases, which are enhanced with formation and accumulation of AGEs [8]. The effect of AGEs is mediated by binding to its receptor, RAGE, which plays an important role in various processes such as aging, renal insufficiency, hypertension, cancer and diabetes [9]. Activation of RAGE leads to oxidative stress, inflammation, angiogenesis, apoptosis and proliferation [10].

Thioredoxin (Trx) is a $12 \mathrm{kD}$ small redox protein known to be present in all organisms. It contains a dithiol-disulfide active site: -Cys-Gly-Pro-Cys-[11], and participates in many cell progress including cell growth, differentiation, and death [12]. Several studies have shown that the decline of Trx expression was found in the development of many diseases including Parkinson's disease (PD), sepsis [13], age-related cataract [14] and DR. In our previous study, we also found that decreased Trx expression plays a key role in retinal neurodegeneration prior to endothelial damage in diabetic mice [15].

We also found that it can delay retina degeneration occurrence after Sulforaphane (SF), Lycium barbarum polysaccharides and grape seed proanthocyanidin extract treatment through up-regulating the expression of Trx in vitro and in vivo [15-17]. Moreover, Previous studies have shown that SF can up-regulate Trx [18] and that SF treatment inhibits retinal degeneration in Tubby mice [19]. It indicated that Trx might play an important role in fighting against the retina degeneration process. Thioredoxin-interacting protein (Txnip, also known as Vitamin D3 Up-regulated Protein 1 (VDUP1), is an endogenous inhibitor of Trx. Studies also suggest that Txnip may play a causal role in cell apoptosis [20]. Previous studies have shown that lack of Txnip expression protects against diabetes and glucotoxicityinduced $\beta$-cell apoptosis [21, 22] and that over-expression of Txnip renders different cell types more susceptible to oxidative stress and apoptosis. Moreover, Txnip inhibition of Trx 


\section{Cellular Physiology Cell Physiol Biochem 2018;50:1673-1686

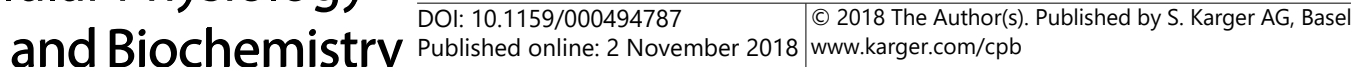 \\ Ren et al.: Trx Inhibited Neurodegeneration}

prevents the anti-proliferative effect of Trx in vascular smooth muscle cells, suggesting a pro-apoptotic function of Txnip [23-25].

Autophagy, specifically macroautopahy, is an important process to maintain cellular homeostasis through lysosomal degradation and recycling of cellular components [26]. The mammalian target of rapamycin (mTOR) is an important regulator of stress-induced autophagy. The phosphatidylinositol 3-kinase (PI3K)- Akt-mTOR signaling pathway is an anti-apoptotic pathway which also plays an essential role in regulating macroautophagy [14]. Interestingly, studies suggest that over-expression of Txnip is associated with reduced mTOR activity [27], whereas knockdown of Txnip using small interfering RNA results in recovery of mTOR activity. Recent evidence suggests that AGEs can trigger autophagy [28]. Although autophagy is typically recognized as a pro-survival mechanism, the consequences of excessive autophagy can lead to cell dysfunction and autophagic cell death, known as type II programmed cell death. Previous studies on the role of autophagy in the pathophysiology of diabetes examined the autophagic process in $\beta$ cells [29]. Additionally, studies have shown that autophagy plays an important role in neurodegenerative disease [30].

Although it is known that oxidative stress, autophagy and apoptosis are associated with DR, little is known about the sequence of events leading to these processes and the specific molecular targets that are associated with DR. In the present study, we used the Neuro 2A cell line and diabetic mouse models to investigate the effect of up-regulation of Trx on AGEinduced apoptosis in vitro and in vivo and to explore the possible mechanisms in order to provide evidence for new clinical therapeutic targets of DR.

\section{Materials and Methods}

\section{Research design}

In vivo, we divided into control and diabetes (DM) groups. In vitro, we used three different methods to up-regulate Trx expression which are SF treatment, stable over-expression Trx, down-regulating TXNIP (an inhibitor of Trx) expression. The experiment groups were divided into control, AGE treatment, AGEand SF-treatment, scramble, shTxnip and shTxnip+AGE treatment in Neuro2A cell and with/without AGE treatment in Neuro2A-LacZ, Neuro2A-Trx cell.

\section{Animal care}

All the experimental procedures were conducted in accordance with institutional guidelines for the care and use of laboratory animals, and protocols were approved by the Institutional Animal Care and Use Committees of Dalian Medical University Laboratory Animal Center. Six-week-old male inbred BALB/c mice weighing 20-25 g (Dalian Medical University Laboratory Animal Center) were housed 5 per cage and subjected to adaptive feed (constant temperature: $22 \pm 2^{\circ} \mathrm{C}, 12$ hours light / 12 hours dark: average illuminance of $80 \mathrm{~lx}$, tap water and food particles). Two weeks later, the mice were randomly divided into non-diabetic group and diabetic group. They were given standard food diets or high glucose and high fat diets (10\% sugar, $10 \%$ lard, $5 \%$ egg yolk, $1 \%$ cholesterol and $0.2 \%$ bile salt) respectively. The diabetic group was injected intraperitonially (IP) with STZ (Sigma) that was dissolved in cold $50 \mathrm{mM}$ citrate buffer (pH 4.5) at a dose of $80 \mathrm{mg} / \mathrm{kg}$ between 10:00 and 14:00. After two injections, the tail vein blood glucose was measured every three days. When the blood glucose reached $\geq 16.7 \mathrm{mmol} / \mathrm{L}$ [31], the mice were considered diabetic and were used for the following experiments.

\section{Electroretinography (ERG)}

Mice were dark adapted overnight and ERG was utilized to assess the function of the retina [15]. Mice were deeply anesthetized with single intraperitoneal injection of ketamine ( $40 \mathrm{mg} / \mathrm{kg}$ ) and xylazine (7 mg/ $\mathrm{kg}$ ). Pupils were dilated with $1 \%$ tropicamide (from Akorn) and $2.5 \%$ phenylephrine (from Akorn). Mice were placed in the center of the dome. ERG (GUOTE, China) system was utilized with light intensity of 10 $\mathrm{cd} \cdot \mathrm{s} / \mathrm{m}^{2}$ and a band pass of $1-300 \mathrm{~Hz}$. The $\mathrm{a}-$ and $\mathrm{b}$ - wave amplitudes were measured in both eyes of the same animal simultaneously. 


\section{Cellular Physiology Cell Physiol Biochem 2018;50:1673-1686 \begin{tabular}{l|l|l} 
and Biochemistry Published online: 2 November 2018 & $\begin{array}{l}\text { (c) } 2018 \text { The Author(s). Published by S. Karger AG, Basel } \\
\text { www.karger.com/cpb }\end{array}$ \\
\hline
\end{tabular}

\section{Morphological analysis}

Morphological analysis was performed as described [32,33]. The mice were euthanized with overdose $\mathrm{CO}_{2}$ and the eyes were removed at the indicated time point and marked with a green dye to orient the superior and inferior retina. Then the eyes were immersed in Bouin's fixative for 24 hours and then in $70 \%$ ethanol for 1-3 days. After dehydration in alcohol, $5-\mu \mathrm{m}$ paraffin sections, containing the entire retina including the optic disc, were made. Sections were then stained with hematoxylin and eosin (H\&E). The number of photoreceptor nuclei was counted in histological sections (5 slices in each group) at $220 \mu \mathrm{m}$ intervals from optic nerve head to both superior and inferior retina by quantification software (Nikon).

\section{Cell culture and reagents}

The Neuro2A cell, obtained from the Institute of Biochemistry and Cell Biology, Chinese Academy of Sciences (Shanghai, China), was cultured in MEM medium (Gibco) containing 10\% fetal bovine serum (Biological industries), penicillin (100 units $/ \mathrm{ml})$, and streptomycin $(100 \mu \mathrm{g} / \mathrm{ml})(\mathrm{HyClone})$ in $5 \% \mathrm{CO}_{2}$ at $37^{\circ} \mathrm{C}$. The medium was replaced every 1 or 2 days. The cells were washed with PBS before the experiments. For treatment with AGEs Bioss (China); catalog number: bs-1158P was utilized. For AGEs: appearance: white powder; source: glycated BSA; purity: $98 \%$. AGEs were dissolved at $20 \mathrm{mg} / \mathrm{ml}$ in MEM medium without fetal bovine serum and stored at $-20^{\circ}$ C. Sulforaphane (SF) was dissolved at $5 \mathrm{mg} / \mathrm{ml}$ in DMSO (Sigma) and stored at $-20^{\circ} \mathrm{C}$.

Plasmid construct and transfection

The cDNA coding for human thioredoxin was amplified from RL-952 cells by reverse transcription polymerase chain reaction using the thioredoxin-specific forward primer: 5'-GGAAGATCTATGGTGAAGCAGATCGAG-3' and the reverse primer: 5'-ACGCGTCGACTTAGACTAATTCATTAATGG-3' (The underlined nucleotides are restriction enzyme sites, Bgl II followed by Sal I ). The PCR products (Bgl II and Sal I restriction) were cloned into the pMD19-T simple cloning vector (Takara) and were confirmed by sequencing. The plasmid was digested by Bgl II and Sal I restriction enzymes (Takara), and then purified by agarose Gel DNA Fragment Recovery Kit Ver.2.0. The thioredoxin gene was produced and inserted into the pIRES2-EGFP expression vector (BD Biosciences, Clontech). In a parallel experiment, LacZ was also inserted into the pIRES2-EGFP expression vector. The resulting recombinant plasmids were named pIRES2-EGFP-Trx and pIRES2-EGFP-LacZ, respectively. These plasmids were stably transfected into Neuro 2A cells. After transient transfection for $24 \mathrm{~h}$, the medium was changed to selection medium containing $400 \mu \mathrm{g} / \mathrm{ml}$ G418 (Sigma). After 4 weeks of selection, several independent clones were picked and were confirmed by RT-PCR, Western blot and immunochemistry.

Sequences of Txnip shRNA and Scramble were pre-designed and synthesized by Takara Bio Incorporated. Txnip shRNA sequences were:

5'-gatccGCCAGCCAACTCAAGAGGCAAAGAAATTCAAGAGATTTCTTTGCCTCTTGAGTTGGCTGGTTTTTTg-3' (sense) and

5'-aattcAAAAAACCAGCCAACTCAAGAGGCAAAGAAATCTCTTGAATTTCTTTGCCTCTTGAGTTGGCTGGCg-3' (anti-sense).

Scramble shRNA sequences were:

5'-gatccGCCACAACAACTGGAGAAACGCGAAATTCAAGAGATTTCGCGTTTCTCCAGTTGTTGTGGTTTTTTg-3' (sense) and

5'-aattcAAAAAACCACAACAACTGGAGAAACGCGAAATCTCTTGAATTTCGCGTTTCTCCAGTTGTTGTGGCg-3' (anti-sense) (Invitrogen, Thermo Fisher Scientific).

Oligonucleotides were diluted in water and annealed by thermal variation. A double strand sequence was then ligated into the vector from the kit and incubated for $14 \mathrm{~h}$ at room temperature with T4 DNA ligase.

Both stable and transit transfection were performed according to the manufacturer's instructions using Lipofectamine2000.

\section{CCK-8 (cell counting kit-8) assay}

The Neuro 2A-Trx and Neuro 2A-LacZ cells were seeded into 96-well plates at the density of $2 \times 10^{6} \mathrm{cells} /$ $\mathrm{ml}$. These cells were treated with different concentrations $(0 \sim 500 \mu \mathrm{g} / \mathrm{ml})$ of AGEs for $6 \mathrm{~h}$. After treatment, cells were incubated with $10 \mu \mathrm{l} \mathrm{CCK-8} \mathrm{reagent} \mathrm{(Dojindo,} \mathrm{Japan)} \mathrm{for} \mathrm{another} 2 \mathrm{~h}$ at $37^{\circ} \mathrm{C}$. The absorbance was read at $450 \mathrm{~nm}$ in a microplate reader (BIO-RAD Model 3550). 


\section{Cellular Physiology Cell Physiol Biochem 2018;50:1673-1686 \begin{tabular}{l|l|l} 
and Biochemistry Published online: 2 November 2018 & $\begin{array}{l}\text { (c) } 2018 \text { The Author(s). Published by S. Karger AG, Basel } \\
\text { www.karger.com/cpb }\end{array}$ \\
\hline
\end{tabular}

\section{Measurement of intracellular ROS}

The formation of intracellular ROS was measured using the DCFH-DA method. Briefly, cells were harvested after being subjected to the various treatments. Cells were washed by PBS for three times, incubated with DCFH-DA probe at a final concentration of $10 \mu \mathrm{mol} / \mathrm{L}$, and then incubated at $37^{\circ} \mathrm{C}$ for another $20 \mathrm{~min}$. Fluorescence was observed by a fluorescent microscope $(200 \times)$.

\section{RT-PCR assay}

Total RNA was extracted from Neuro 2A and Neuro 2A-Trx/LacZ cells in TRIZOL (Takara) according to the manufacturer's instructions. The cDNA was synthesized from 500ng of RNA in a $10 \mu$ reaction with RNA PCR Kit (AMV) Ver.3.0 (Takara). GAPDH was used as a housekeeping loading control. The primers used were: Trx (human) forward 5'-GGAAGATCTATGGTGAAGCAGATCGAG-3', reverse 5'- ACGCGTCGACTTA GACTAATTCATTAATGG-3', LacZ forward 5'-GGAAGATCTGTCGTTTTACAACGTCGT-3', reverse 5'-ACGCGTCGACTTTTTGACACCAGACCAAC-3', Txnip forward 5'-ACTCCTCAAGATGGGTGGCAATC-3', reverse 5'-ACATCCACCCAGCAAACACTCCT-3', GAPDH forward 5'-TGTGATGGGTGTGAACCACGAGAA-3' reverse 5'-GAGCCCTTCCACAATGCCAAAGTT-3'. PCR amplification conditions were as follows: $94^{\circ} \mathrm{C}$ for 2 minutes, 30 cycles of: $94^{\circ} \mathrm{C}$ for 30 seconds, $55^{\circ} \mathrm{C}$ for 30 seconds, and $68^{\circ} \mathrm{C}$ for 3 minutes, and a final extension $72^{\circ} \mathrm{C}$ for 10 minutes. The PCR products were run on a $1 \%$ agarose gel at $120 \mathrm{~V}$ for 30 minutes and stained with ethidium bromide (EB). The data was analyzed with a GDS-8000 Bio imaging system (UVP, Upland, CA) and Lab Works 4.5 software (UVP).

\section{Western Blot analysis}

Cells were lysed in a buffer containing $50 \mathrm{mM}$ Tris- $\mathrm{HCl}$ pH 8.0, 150mM NaCl, 1\% Nonidet P-40, $0.5 \%$ deoxycholate, $0.1 \%$ SDS, $1 \mathrm{mM}$ PMSF and 150 units $/ \mathrm{ml}$ aprotinin on ice. After centrifugation, cell extracts were resolved on SDS-PAGE and electroblotted onto polyvinylidenedifluoride (PVDF) membranes (Millipore, Billerica, MA). After blocking with 5\% non-fat milk in TTBS, the membranes were probed with antibodies. Primary antibody incubations were performed at the following dilutions: AGEs (Abcam, ab23722, 1:1000), Trx (Cell Signaling Technology, 24291:1000), Trx (human) (Abcam, ab133524, 1:5000), Txnip (Cell Signaling Technology, \#14715, 1:1000), LC3B (Proteintech, 12135-1-AP, 1:1000), and actin (Santa Cruz, sc-47778, 1:1000). All incubations were carried out overnight at $4^{\circ} \mathrm{C}$. The membranes were then washed three times with $1 \times$ TTBS for 5 minutes. Subsequently, the membranes were incubated with goat anti-rabbit IgG (Abclonal, AS014, 1:1000) and goat anti-mouse IgG (Abclonal, AS003, 1:1000) for 1 hour at room temperature and washed 3 times with $1 \times$ TTBS for 15 minutes. The membranes were then exposed to X-ray film using an enhanced chemiluminescence system. The intensities of the bands were measured using LabWorks 4.5 .

\section{TUNEL assay}

Neuro 2A-LacZ and Neuro 2A-Trx cells were plated onto the cover slip and cultured overnight, and then were with different treatments. The cells were washed twice with PBS, resuspended in 50 $\mathrm{\mu l} /$ well TUNEL reaction mixture (for the negative control add $50 \mu \mathrm{l}$ Label solution), and incubated for $60 \mathrm{~min}$ at $37^{\circ} \mathrm{C}$ in a humidified atmosphere without light exposure. Samples were then washed twice in PBS and imaged using a fluorescence microscope $(200 \times)$.

\section{Flow Cytometry Analysis}

The apoptotic incidence was detected by Annexin V/propidium iodide (PI), Annexin V-APC/7-AAD and binding buffer (KeyGEN Biotech). Neuro 2A cells were treated with various treatments. Cells were then collected and re-suspended in $500 \mu \mathrm{l} 1$ xbinding buffer and then incubated for 15 minutes without light exposure. Apoptotic cells, including early apoptotic (Annexin $\mathrm{V}^{+} / \mathrm{PI}^{-}$) and late apoptotic/necrotic (Annexin $\mathrm{V}^{+} / \mathrm{PI}^{+}$) cells, were counted using FACS (ACEA Biosciences, CA, USA).

\section{Transmission electron microscopy}

Neuro 2A-LacZ and Neuro 2A-Trx cells were treated with or without 100 $\mu \mathrm{g} / \mathrm{ml}$ AGEs for 6 hours and washed twice with PBS before harvesting and were then fixed in $2 \%$ glutaraldehyde. Ultrathin slices were prepared and observed under the JEM-2000EX* transmission electron microscope. 


\section{Cellular Physiology Cell Physiol Biochem 2018;50:1673-1686 \begin{tabular}{ll|l} 
and Biochemistry & $\begin{array}{l}\text { DOI: 10.1159/000494787 } \\
\text { Published online: 2 November } 2018\end{array}$ & $\begin{array}{l}\text { (c) } 2018 \text { The Author(s). Published by S. Karger AG, Basel } \\
\text { www.karger.com/cpb }\end{array}$
\end{tabular} \\ Ren et al:: Trx Inhibited Neurodegeneration}

\section{Immunofluorescence}

Cells were seeded on coverslips 24 hours prior to treatment. After treatment with AGEs for 6 hours, the cells were fixed in $4 \%$ formaldehyde solution. Next, the cells were permeabilized with $0.5 \%$ TritonX-100 (Sigma-Aldrich, USA) and blocked with 5\% BSA (Solarbio, USA). Subsequently, the cells were incubated with Txnip (Cell Signaling Technology, \#14715, 1:100) and p-mTOR (Cell Signaling Technology, \#5536, 1:100) overnight at $4^{\circ} \mathrm{C}$. After thorough washing with PBS, cells were incubated with Alexa Fluor 555 Donkey AntiRabbit IgG (Heavy + Light chains) (A-31572, Invitrogen, USA) solution for 1.5 hours at room temperature. The nuclei were stained with DAPI. Coverslips were mounted on the slides with a drop of mounting medium (H-1000, Vector, USA). Images were taken using a fluorescence microscope (Nikon Ti-S, Japan) $(400 \times)$.

\section{SF treatment}

In vitro, the Neuro $2 \mathrm{~A}$ cells were treated with SF for 6 hours and 12 hours at $0.5 \mu \mathrm{M}$ and then were prepared for the various experiments.

\section{Statistical analysis}

The data were presented using mean \pm SD and analyzed by Student's t-test (two groups) and one-way analysis of variance (ANOVA) (three or more groups). The data are presented as the mean \pm SD from three independent experiments.

All statistical analysis were made using GraphPad Prism (Ver.5.0) and SPSS 17.0 software. A $p$ value of $<0.05$ was considered significant.

\section{Results}

Up-regulation of thioredoxin inhibited retinal neurodegeneration in diabetic mice

After establishing our mouse model of diabetes, we used ERG to assess the function of the retina. The a-wave, corresponding the photoreceptor function, and the b-wave, corresponding to inner retinal function, were decreased about 50\% in DM group compared with control group (Fig. 1A). We isolated the mouse retinas and used H\&E staining to observe the retinal morphology and measure retinal thickness. The retinal thickness was defined as

Fig. 1. Retina neuronal cell degeneration and related factors in diabetic mice. (A) The function of the retina was detected by ERG in diabetic mice. The $a$ and $b$ waves all decreased in diabetes mice. (B) Morphology of retina neuronal cells were observed by H\&E staining in diabetic mice. The thickness was decreased in diabetes mice.

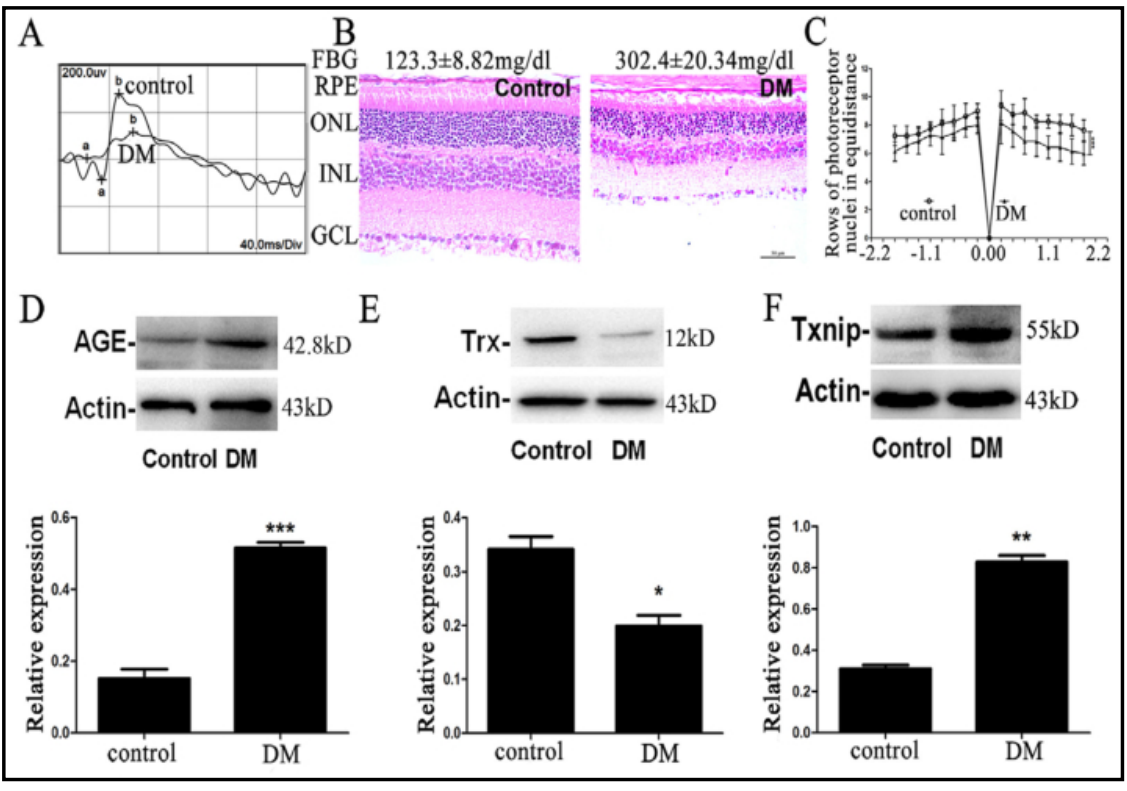

(C) The nuclear counting of outer nuclear layer in diabetic mice. The number of nuclei was decreased in diabetes mice. The protein expression of AGE (D), Trx (E) and Txnip (F) in diabetic mice were analyzed by Western blot. ( $n=5$ in each group) ${ }^{*} \mathrm{p}<0.05,{ }^{* *} \mathrm{p}<0.01,{ }^{* * *} \mathrm{p}<0.001$. 


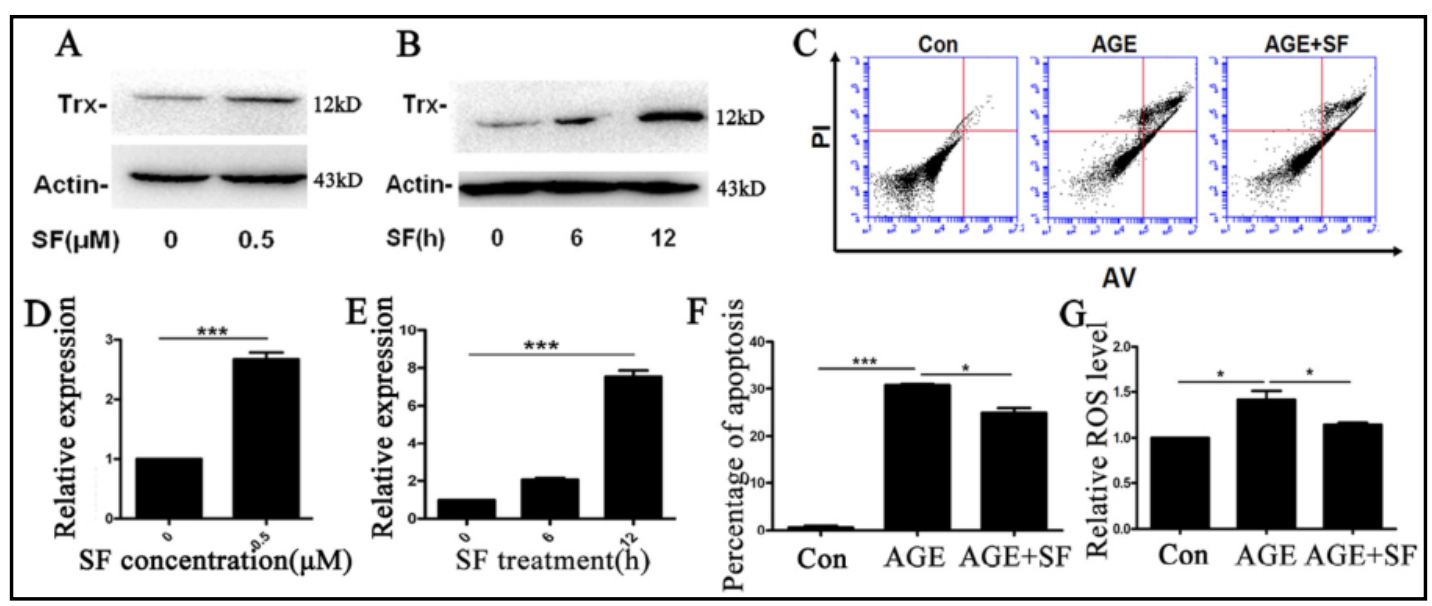

Fig. 2. The effect of up-regulating Trx expression by SF on the apoptosis induced by advanced glycation end products (AGEs). The expression of Trx was analyzed by Western blot after treatment with different concentration (A\&D) and time (B\&E) of SF. (C\&F) Apoptosis of Neuro 2A cell was analyzed by flow cytometry with SF and AGE treatments. (G) AGE-induced production of ROS in Neuro2a cells after SF treatment, as detected by a DCFH-DA probe. ( $\mathrm{n}=3$ in each group) ${ }^{*} \mathrm{p}<0.05$, ${ }^{* * *} \mathrm{p}<0.001$.

the distance from the retinal pigment epithelium (RPE) layer to the ganglion cell layer (GCL). As shown in Fig. 1B\&C, retinal thickness was reduced in the DM group as compared to the control group. To examine the levels of AGEs, Trx and Txnip in the DM and control group we used Western blot. The expression of AGEs and Txnip was increased in the DM group compared to the control group (Fig. 1D\&F) $(p<0.01)$. Interestingly, the expression of Trx was decreased in the DM group compared with control group (Fig. 1E) $(p<0.05)$.

The protective effect of up-regulation of Trx by SF on the AGE-induced apoptosis in vitro

In vitro, Neuro $2 \mathrm{~A}$ cells were treated with $0.5 \mu \mathrm{M}$ SF for 6 or $12 \mathrm{~h}$. The Trx expression was increased after SF treatment as detected by Western bolt (Fig. 2A-B\& D-E) $(p<0.001$, $p<0.001)$. AGE-induced apoptosis was analyzed in Neuro 2A cells by flow cytometry with or without SF treatment. As shown in Fig. 2C\&F, the percentage of apoptotic Neuro 2A cells increased after treatment with AGEs as compared with control group $(p<0.001)$. However, the percentage of apoptotic Neuro 2A cells was reduced in the group treated with AGEs and $\mathrm{SF}$ compared with the group treated with AGEs alone $(p<0.05)$. ROS production in the AGEtreated group was increased compared with the control group, however, it can be decreased after SF treatment $(p<0.05)$ (Fig. 3G).

Stable over-expression Trx inhibit apoptosis AGE-induced in Neuro $2 A$ cell

To investigate the influence of Trx over-expression on apoptosis in Neuro 2A cells, we established stable Neuro 2A cell line that stably over-expresses Trx and a parallel control group that stably over-expresses LacZ (Fig. 3A). RT-PCR, immunocytochemistry and Western blot were used to confirm the stable cell lines (Figures 3B-D).

We also used the CCK-8 assay to detect the viability of the Neuro 2A cells after treatment with AGEs. The Neuro 2A cells were treated with AGEs at different concentrations $(0,100$, $200,300,400,500 \mu \mathrm{g} / \mathrm{ml}$ ) for 6 hours. As shown in Fig. 3E, the cell viability was significantly decreased in the Neuro 2A-LacZ cell line, however, the cell viability remained unchanged in the Neuro 2A-Trx cells after treatment with AGEs (Fig. 3E) $(p<0.05)$.

Intracellular reactive oxygen species (ROS) play a critical role in different types of cell survival. ROS generation was detected after treatment with AGEs in the Neuro 2A cell lines. After treatment with AGEs, ROS generation was increased in the Neuro 2A-LacZ cells, however, it was attenuated by over-expression Trx in the Neuro 2A-Trx cells (Fig. 3F). We also evaluated the AGE-induced apoptosis on Neuro 2A-Trx/LacZ cells by TUNEL staining. The 


\section{Cellular Physiology Cell Physiol Biochem 2018;50:1673-1686 and Biochemistry DOl: 10.1159/000494787 2018 (0) 2018 The Author(s). Published by S. Karger AG, Basel

Fig. 3. The effect of stable over-expression of Trx on apoptosis induced by AGEs in Neuro 2A cell. (A) Transfection efficiency was observed in Neuro 2A cell at different time. (B) The expression of Trx and LacZ was detected in different clone in Neuro 2A cell by RTPCR. (C) The expression of Trx (human) were detected by immunocytochemical staining (Green fluorescence) and the nuclei was stained by DAPI (blue). (D) The expression of Trx (human) and EGFP was detected by Western blot. (E) The viability of Neuro 2A-Trx/LacZ cells were measured by CCK-8 assay at different concentration $\quad(0 \sim 500 \mu \mathrm{g} /$ $\mathrm{ml}$ ) of AGE. (F) Intracellular ROS was detected by DCFHDA (Green fluorescence) and the nuclei was stained by DAPI (blue) in Neuro 2A-Trx/LacZ after AGEs treatment. Magnification, 200x. (G) Apoptosis was detected by TUNEL staining (Green fluorescence) and the nuclei was stained by DAPI(blue) in Neuro 2A-Trx/LacZ cells after AGEs treatment. Magnification, 200x. (H) The expression of Txnip was detected in Neuro 2A-Trx/LacZ cells after

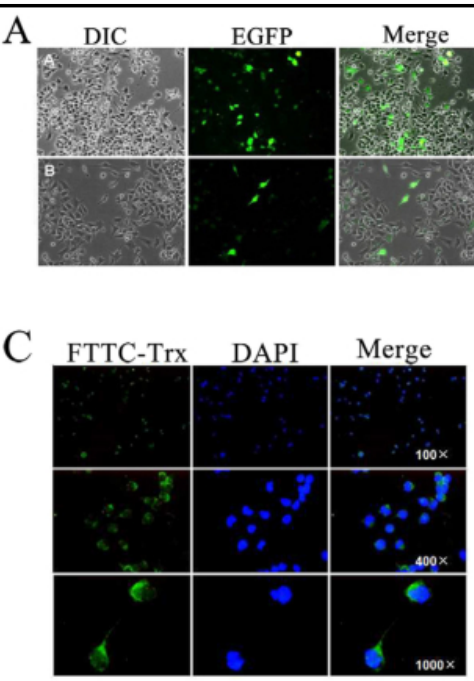

$\mathrm{E}$
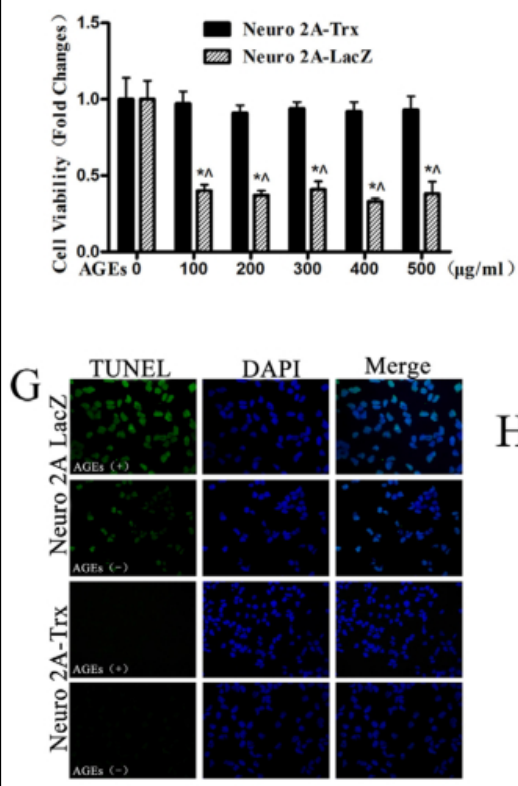

$\mathrm{H}$

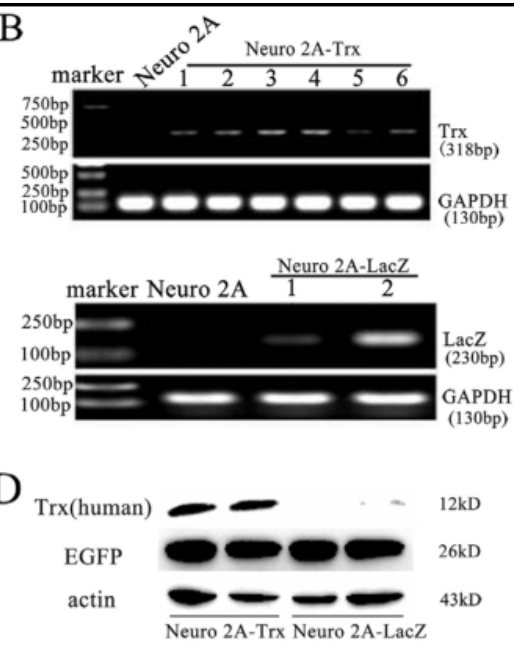

F
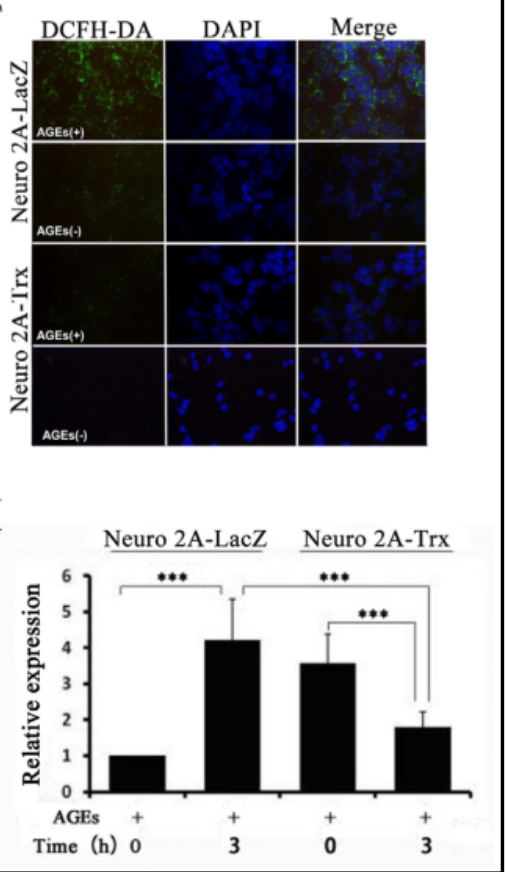

AGEs treatment. $(\mathrm{n}=3$ in

each group) ${ }^{*} \mathrm{p}<0.05, \# \mathrm{p}<0.05,{ }^{* * *} \mathrm{p}<0.001$. *: compare with $\mathrm{NC}(\mathrm{AGEs}=0 \mu \mathrm{g} / \mathrm{ml})$ group, \#: compare with the Neuro 2A-Trx group at the same concentration.

results showed that AGEs treatment could significantly increase apoptosis in Neuro 2A-LacZ cells. However, apoptosis due to treatment with AGEs was inhibited in the Neuro 2A-Trx cells (Fig. 3G). Levels of Txnip expression were measured after AGEs treatment in the Neuro 2A cell lines using qRT-PCR. The expression of Txnip was increased in the Neuro 2A-LacZ cells and it was suppressed in Neuro 2A-Trx cells after treatment with AGEs (Fig. 3H) $(p<0.001)$.

Up-regulation of Trx through inhibition of Txnip prevents AGE-induced apoptosis in Neuro 2 A cells

Txnip is an endogenous inhibitor of Trx. To decrease Txnip expression, we used a gene silencing expression vector (shRNA Txnip). shRNA Txnip was constructed and transfected into Neuro 2A-LacZ. The transfection efficiency was observed at various time points after 


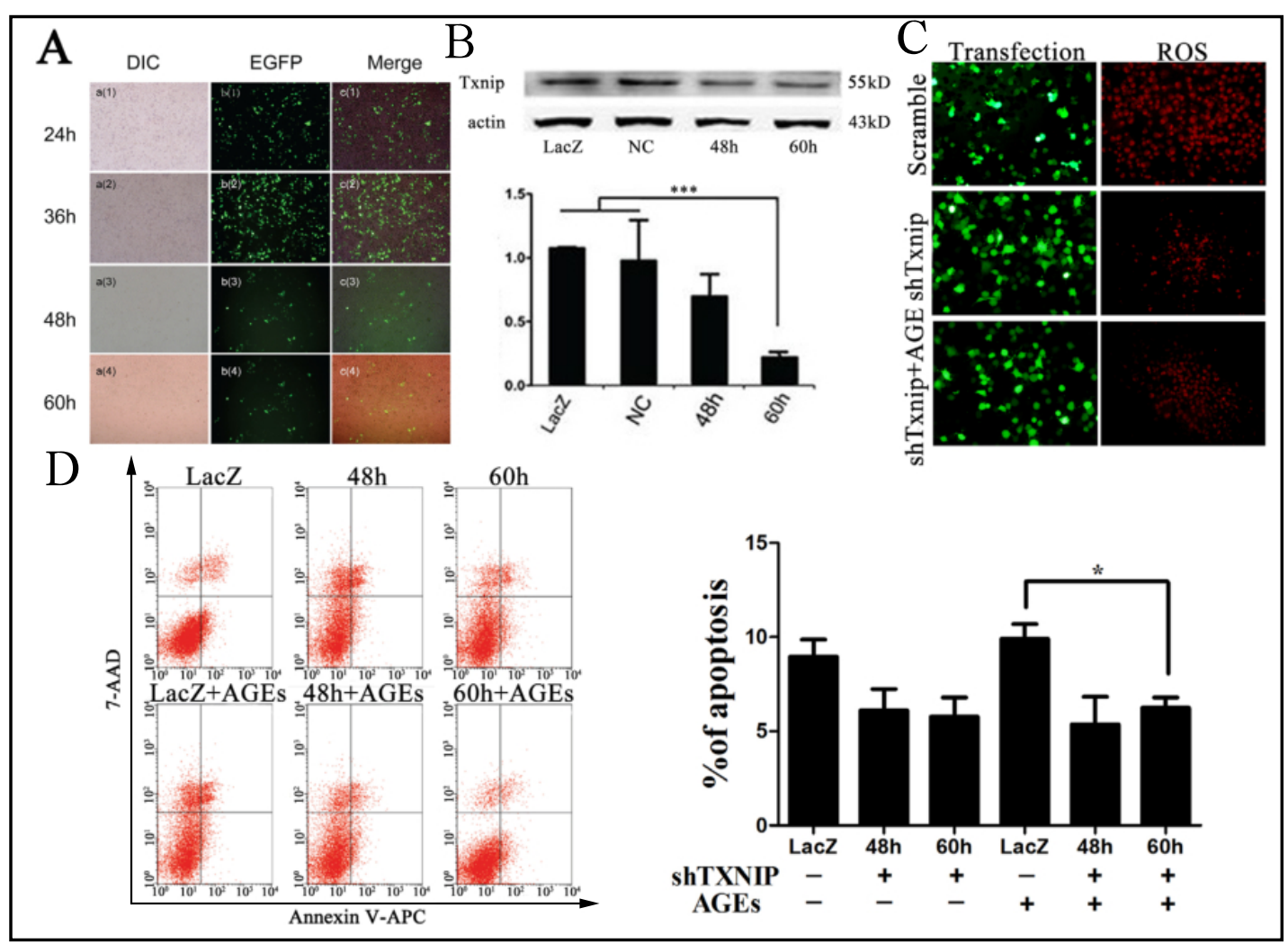

Fig. 4. The effect of up-regulating Trx expression by down-regulating Txnip on apoptosis induced by AGEs. (A) Transfection efficiency observation in Neuro 2A at different time (24 h, 36 h, 48 h, 60 h). Magnification, 200x. (B) The expression of Txnip were analyzed by Western blot after transfected with Txnip-shRNA at different time. (C) The effect of up-regulating Trx expression by down-regulating Txnip on intracellular ROS (Red fluorescence) in Neuro 2A-LacZ cell. Magnification, 200x. (D) Apoptosis of Neuro 2A-LacZ cell was analyzed by flow cytometry after transfected shRNA-Txnip with/without AGEs treatment. ( $n=3$ in each group) ${ }^{*}$ p $<0.05, * * * p<0.001$.

transfection ( 24 hours, 36 hours, 48 hours, and 60 hours) by fluorescence microscope. The expression of Txnip was measured by Western blot after transfection (Fig. 4A\&B) $(p<0.001)$. ROS generation was measured after down-regulation of Txnip in Neuro 2A-LacZ cell after AGEs treatment. As shown in Fig. 4C, after treatment with AGEs, ROS levels were reduced in Neuro 2A-LacZ cells transfected with shRNA Txnip. Additionally, the percentage of apoptotic cells was quantified in Neuro 2A-LacZ cells using flow cytometry. After treatment with AGEs, the percent apoptotic cells is reduced in the cells treated with shRNA Txnip as compared with those transfected with a scrambled shRNA (Fig. 4D) $(p<0.05)$.

\section{Over-expression Trx suppresses AGE-induced autophagy in Neuro $2 A$ cells}

To investigate the influence of over-expression of Trx on autophagy in Neuro 2A cells we used transmission electron microscopy (TEM). Cells were treated with or without 100 $\mu \mathrm{g} / \mathrm{ml}$ AGEs for 6 hours before collection for TEM. Autophagosomes are morphological hallmarks of autophagy. The TEM analyses showed a greater number of single and doublemembrane vesicles accumulated in control cells, Neuro 2A-LacZ, as compared to Neuro 2A-Trx cells (Fig. 5A). Quantification of these vesicles confirmed these observations (Fig. $5 \mathrm{~B})(p<0.005)$. Treatment with AGEs led to accumulation of autophagosomes in the Neuro 2A-LacZ cells (Fig. 5B) $(p<0.05)$. LC3-II is a key marker of autophagy, and Western blot analysis showed that over-expression Trx could attenuate up-regulation of LC3-II induced by treatment with AGEs (Fig. 5C) $(p<0.05)(p<0.005)$. Then, we used immunofluorescence to detect the expression of Txnip and p-mTOR. We found that over-express Trx could reduce 


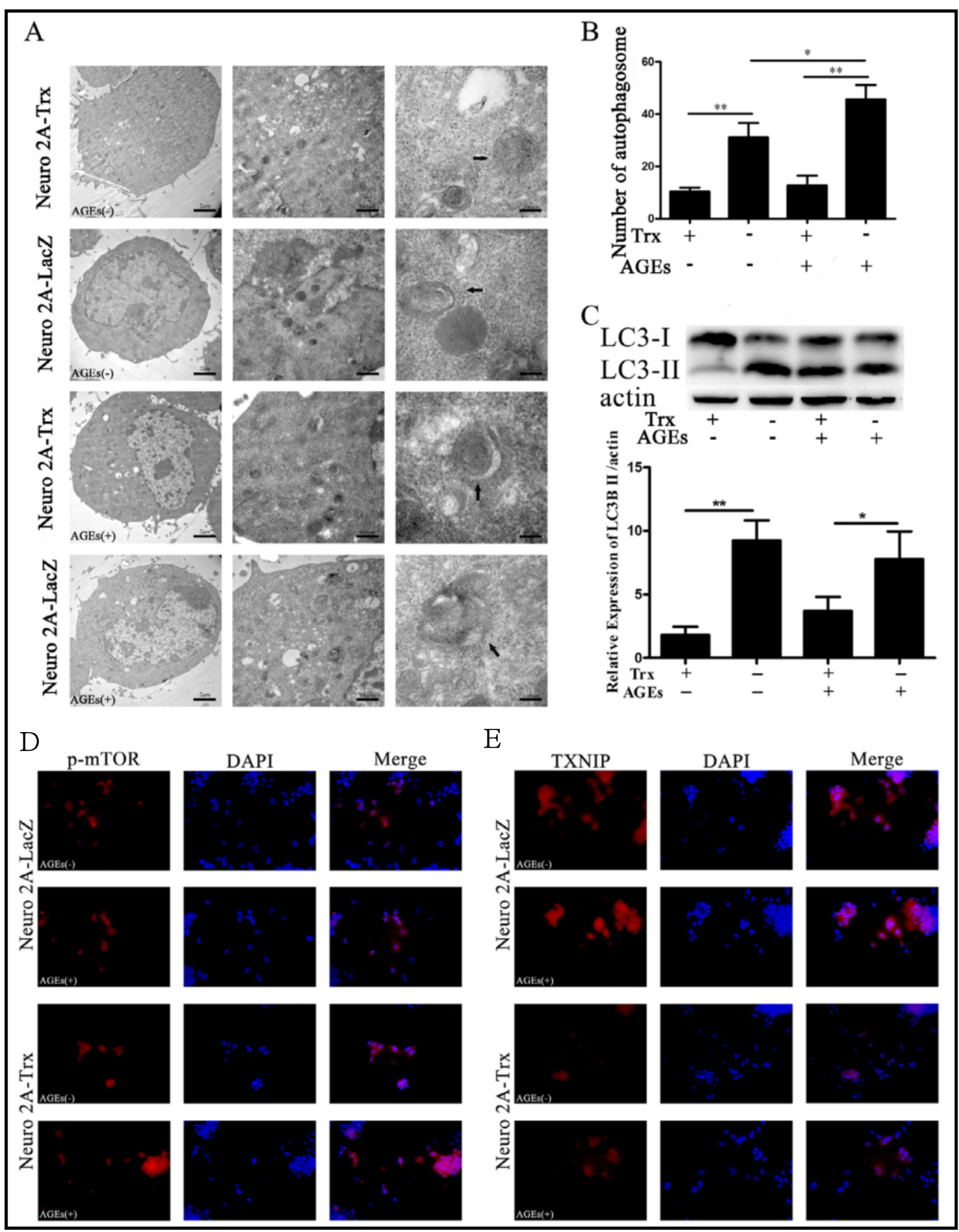

Fig. 5. Over-expression of Trx suppressed AGE-induced autophagy in Neuro 2A cell. (A) Autophagosomes of Neuro 2A-Trx/LacZ cells were observed by transmission electron microscopy after treated with/without AGEs, and the number counting of autophagosomes in each cell (B). (C) The expression of LC3B was analyzed by Western blot. The expression of p-mTOR (Red fluorescence) (D), TXNIP (Red fluorescence) (E) and the nuclei was stained by DAPI (blue) was analyzed by immunofluorescence after treated with/without AGEs. Magnification, 400x. ( $\mathrm{n}=3$ in each group) ${ }^{*} \mathrm{p}<0.05$, ${ }^{* *} \mathrm{p}<0.01$.

the expression of Txnip and activite p-mTOR compare with Neuro 2A-LacZ cells. However, after treatment with AGEs, the expression of Txnip in Neuro 2A-LacZ was increased but it was unchanged in Neuro 2A-Trx cells (Fig. 5D). As shown in Fig. 5E, there are similar results 


\section{Cellular Physiology Cell Physiol Biochem 2018;50:1673-1686 \begin{tabular}{ll|l} 
and Biochemistry & $\begin{array}{l}\text { DOl: 10.1159/000494787 } \\
\text { Published online: } 2 \text { November } 2018\end{array}$ & $\begin{array}{l}\text { (2018 The Author(s). Published by S. Karger AG, Basel } \\
\text { www.karger.com/cpb }\end{array}$ \\
\cline { 2 - 3 }
\end{tabular} \\ Ren et al.: Trx Inhibited Neurodegeneration}

that the activation of p-mTOR were decreased in Neuro 2A-LacZ cells after treatment with AGEs but unchaged in Neuro 2A-Trx cells.

\section{Discussion}

Diabetes retinopathy (DR) is a leading cause of vision loss in middle-aged and elderly people globally [1]. Studies have shown that there are many risk factors for DR such as hyperglycemia, hypertension, hyperlipidemia, Body Mass Index (BMI), puberty and pregnancy [34].

AGEs refers to a group of stable end products produced by the Maillard reaction series of free amino groups of proteins, amino acids, lipids or nucleic acids, as well asaldehyde groups of glucose or other reducing sugars, under non-enzymatic conditions [24]. Accumulation of AGEs is found in many neurodegenerative diseases [8]. Our previous study confirmed that neurodegeneration in diabetic retinopathy precedes vascular lesions [15] and several studies have demonstrated that diabetic retinopathy is a neurodegenerative disorder [5]. AGEs are more reactive than sugars because AGEs receptors are widely distributed in various tissue cells such as endothelial cells, smooth muscle cells, and immune system cells. AGEs can mediate their effects via specific receptors, such as the receptor for AGE (RAGE) and activate diverse signal transduction cascades and downstream pathways. Binding of AGEs to their receptors can trigger several age-related [9] and diabetes-related diseases, as well as cardiovascular diseases [7]. AGE-RAGE interaction activates nuclear factor (NF)$\kappa \mathrm{B}$ transducing a variety of inflammatory and innate immune responses in the cell. In this study, accumulation of AGEs was found in the retinas of diabetic mice, which confirms that the accumulation of AGEs plays an important role in neurodegenerative diseases. In the process, we also found that the expression of Trx, an antioxidant small molecule protein, was decreased, demonstrating that Trx may play a key role in diabetic retinopathy pathogenesis.

The main mechanism by which AGEs induce apoptosis is through accumulation of ROS which oxidize Trx. The Trx system is responsible for protecting cells from oxidative stress, and is composed of several related molecules that form a network of interactions, and use cysteine residues in their active site to combat oxidative species [11, 13, 23]. Sulforaphane(SF), which is a naturally occurring isothiocyanate compound isolated from cruciferous vegetables, upregulates Trx [19]. In vivo, we found that the expression of Trx increased in a dose-dependent manner with increased SF concentration. This corresponded with increased retinal thickness in diabetic mice. We also used SF to regulate Trx expression in vitro and found that the same effect: when the intracellular Trx expression increased, the apoptosis decreased.

Trx exerts its role through interaction with its binding proteins [21]. One of the most important proteins is apoptosis signal-regulating kinase 1 (ASK1), an important regulator of apoptosis. When Trx is oxidized in response to oxidative stress, ASK1 is dissociated from the oxidized Trx and activated to induce an apoptotic signal. Trx also interacts with thioredoxin interacting protein (Txnip)[20]. Txnip is considered a pro-oxidative stress and pro-apoptotic protein under conditions of diabetes and cellular stress. Our present studies have shown that AGE exposure induces extensive expression of Txnip in a time-dependent manner. However, when Trx is over-expressed in the Neuro 2A cell line, Txnip expression does not increase after treatment with AGEs. Here we have successfully shown that over-expression of Trx is able to inhibit cell apoptosis induced by AGEs. Some studies have shown that ROS can inhibit cell viability and proliferation and play an important role in promoting apoptosis [35]. When we up-regulated Trx indirectly, through inhibition of Txnip using shRNA, the generation of intracellular ROS was significantly decreased and the cells were protected from apoptosis.

AGEs, a harmful substance accumulated in the retina of diabetic mice [8], caused cellular apoptosis in vitro. It is also known that AGEs can induce autophagy in cells and that excessive autophagy can cause cell death $[28,36]$. Several studies have confirmed that several signaling pathways, such as the PI3K / AKT / mTOR signaling pathway [37], p38- 


\section{Cellular Physiology Cell Physiol Biochem 2018;50:1673-1686 and Biochemistry DOl: 10.1159/000494787, 2018 The Author(s). Published by S. Karger AG, Basel

MARK pathway [38] and ERKrelated signaling pathway [39], are associated with autophagy. Studies have shown that excessive autophagy can cause cell dysfunction and autophagic cell death [40]. The mammalian target of rapamycin (mTOR) is an important protein in the autophagy signaling pathway and is known to drive the onset of a range of human diseases. In our study, we found that it can induce AGE accumulation in diabetes in mice which can lead to retina degeneration with the Trx down-regulating and upregulating Txnip. Moreover, we have demonstrated that AGEs induce ROS generation, activate autophagy, and lead to eventual apoptosis. Several studies have demonstrated that Trx and its endogenous inhibitor Txnip help sustain the cellular redox balance in response to various stresses and both play a crucial role in cell proliferation and growth [12]. We used different methods to up-regulate Trx expression and confirmed that over-expression Trx or downregulation Txnip can decrease intracellular ROS generation and apoptosis. Moreover, we found that over-expression of Trx is able to inhibit AGE-induced autophagy which may be related to inhibition of the Txnip/mTOR pathway. Finally, we have summarized the underlying mechanism in the Fig. 6.

AGE-induced cell apoptosis is regulated by Trx. We have shown that Trx expression decreases after AGEs treatment in Neuro 2A cells. Up-regulation of Trx reduces ROS generation and protects cells from apoptosis induced by AGEs. During this process Trx may inhibit autophagy though the Txnip and mTOR pathway.

\section{Conclusion}

Up-regulating thioredoxin expression inhibits neurodegeneration induced by AGEs. The underlying mechanism may be related to inhibition of the Txnip/mTOR pathway mediated autophagy.

\section{Acknowledgements}

This work was supported by Grant No. 31371218 from the National Natural Science Foundation of China, Grant No. LQ2017005 from the Basic Scientific Research Projects of Liaoning provincial education department. This work was also supported by Liaoning Provincial Program for Top Discipline of Basic Medical Sciences. 


\section{Cellular Physiology Cell Physiol Biochem 2018;50:1673-1686 \begin{tabular}{ll|l} 
and Biochemistry & $\begin{array}{l}\text { DOI: 10.1159/000494787 } \\
\text { Published online: 2 November } 2018\end{array}$ & $\begin{array}{l}\text { (c) } 2018 \text { The Author(s). Published by S. Karger AG, Basel } \\
\text { www.karger.com/cpb }\end{array}$
\end{tabular} \\ Ren et al.: Trx Inhibited Neurodegeneration}

\section{Disclosure Statement}

The authors declare to have no competing interests.

\section{References}

$>1$ Wild S RG, Green A, Sicree R, King H: Global prevalence of diabetes: estimates for the year 2000 and projections for 2030. Diabetes Care 2004;27:53.

-2 Kim J, Cheon H, Jeong YT, Quan W, Kim KH, Cho JM, Lim YM, Oh SH, Jin SM, Kim JH, Lee MK, Kim S, Komatsu M, Kang SW, Lee MS: Amyloidogenic peptide oligomer accumulation in autophagy-deficient beta cells induces diabetes. J Clin Invest 2014;124:3311-3324.

- Kahn SE: The relative contributions of insulin resistance and beta-cell dysfunction to the pathophysiology of Type 2 diabetes. Diabetologia 2003;46:3-19.

4 Muriach M, Flores-Bellver M, Romero FJ, and Barcia JM: Diabetes and the brain: oxidative stress, inflammation, and autophagy. Oxid Med Cell Longev 2014;2014:102158.

5 Yang Q Xu Y, Xie P, Cheng H, Song Q, Su T, Yuan S, Liu Q: Retinal Neurodegeneration in db/db Mice at the Early Period of Diabetes. J Ophthalmol 2015;2015:757412.

6 Federation ID: Diabetes atlas. 2015;6thed, Brussels, Belgium.

7 Chawla D, Bansal S, Banerjee BD, Madhu SV, Kalra OP, Tripathi AK: Role of advanced glycation end product (AGE)-induced receptor (RAGE) expression in diabetic vascular complications. Microvasc Res 2014;95:1-6.

8 Mastrocola R: AGEs and neurodegeneration: the Nrf2/glyoxalase-1 interaction. Oncotarget 2017;8:56455646.

-9 Senatus LM, Schmidt AM: The AGE-RAGE Axis: Implications for Age-Associated Arterial Diseases. Front Genet 2017;8:187.

10 Hollenbach M: The Role of Glyoxalase-I (Glo-I), Advanced Glycation Endproducts (AGEs), and Their Receptor (RAGE) in Chronic Liver Disease and Hepatocellular Carcinoma (HCC). Int J Mol Sci DOI:10.3390/ ijms18112466.

11 Zeng XS, Jia JJ, Kwon Y, Wang SD, Bai J: The role of thioredoxin-1 in suppression of endoplasmic reticulum stress in Parkinson disease. Free Radic Biol Med 2014;67:10-18.

12 Lu J, Holmgren A: Thioredoxin system in cell death progression. Antioxid Redox Signal 2012;17:1738-1747.

13 Chen G, Li X, Huang M, Li M, Zhou X, Li Y, Bai J: Thioredoxin-1 Increases Survival in Sepsis by Inflammatory Response Through Suppressing Endoplasmic Reticulum Stress. Shock 2016;46:67-74.

14 Zhou J, Yao K, Zhang Y, Chen G, Lai K, Yin H, Yu Y: Thioredoxin Binding Protein-2 Regulates Autophagy of Human Lens Epithelial Cells under Oxidative Stress via Inhibition of Akt Phosphorylation. Oxid Med Cell Longev 2016;2016:4856431.

15 Ren X, Li C, Liu J, Zhang C, Fu Y, Wang N, Ma H, Lu H, Kong H, Kong L: Thioredoxin plays a key role in retinal neuropathy prior to endothelial damage in diabetic mice. Oncotarget 2017;8:61350-61364.

16 Ren X, Lu H, Wang N, Zhang C, Ji Y, Cui S, Dong Y, Yang K, Du M, Diao F, and Kong L: Thioredoxin is implicated in the antiapoptotic effects of grape seed proanthocyanidin extract during hyperglycemia. Mol Med Rep 2017;16:7731-7737.

-17 Tang L, Bao S, Du Y, Jiang Z, Wuliji AO, Ren X, Zhang C, Chu H, Kong L, Ma H: Antioxidant effects of Lycium barbarum polysaccharides on photoreceptor degeneration in the light-exposed mouse retina. Biomed Pharmacother 2018;103:829-837.

$>18$ Li Z, Galli U, Becker LE, Bruns H, Nickkolgh A, Hoffmann K, Karck M, and Schemmer P: Sulforaphane protects hearts from early injury after experimental transplantation. Ann Transplant 2013;18:558-566.

19 Kong L, Liu B, Zhang C, Wang B, Wang H, Song X, Yang Y, Ren X, Yin L, Kong H, Ma H: The therapeutic potential of sulforaphane on light-induced photoreceptor degeneration through antiapoptosis and antioxidant protection. Neurochem Int 2016;100:52-61.

20 Devi TS, Lee I, Huttemann M, Kumar A, Nantwi KD, Singh LP: TXNIP links innate host defense mechanisms to oxidative stress and inflammation in retinal Muller glia under chronic hyperglycemia: implications for diabetic retinopathy. Exp Diabetes Res 2012;2012:438238. 


\section{Cellular Physiology Cell Physiol Biochem 2018;50:1673-1686 and Biochemistry \begin{tabular}{c|c|c|c|} 
DOI: 10.1159/000494787 \\
Publisher 2018 The Author(s). Published by S. Karger AG, Basel
\end{tabular}

21 Chen J, Saxena G, Mungrue IN, Lusis AJ, Shalev A: Thioredoxin-interacting protein: a critical link between glucose toxicity and beta-cell apoptosis. Diabetes 2008;57:938-944.

22 Junqin Chen GF, Geetu Saxena: Lack of TXNIP Protects Against Mitochondria-Mediated. DIABETES 2010;59:440-447.

-23 Altschmied J, Haendeler J: Thioredoxin-1 and endothelial cell aging: role in cardiovascular diseases. Antioxid Redox Signal 2009;11:1733-1740.

-24 Li Y, Chang Y, Ye N, Dai D, Chen Y, Zhang N, Sun G, and Sun Y: Advanced Glycation End Products Inhibit the Proliferation of Human Umbilical Vein Endothelial Cells by Inhibiting Cathepsin D. Int J Mol Sci DOI: 10.3390/ijms18020436.

-25 Liu Z, Sun M, Wang Y, Zhang L, Zhao H, Zhao M: Silymarin attenuated paraquat-induced cytotoxicity in macrophage by regulating Trx/TXNIP complex, inhibiting NLRP3 inflammasome activation and apoptosis. Toxicol In vitro 2018;46:265-272.

26 Levine B, Kroemer G: Autophagy in the pathogenesis of disease. Cell 2008;132:27-42.

27 Jin HO, Seo SK, Kim YS, Woo SH, Lee KH, Yi JY, Lee SJ, Choe TB, Lee JH, An S, Hong SI, Park IC: TXNIP potentiates Redd1-induced mTOR suppression through stabilization of Redd1. Oncogene 2011;30:37923801.

28 Sun K, Wang W, Wang C, Lao G, Liu D, Mai L, Yan L, Yang C, Ren M: AGEs trigger autophagy in diabetic skin tissues and fibroblasts. Biochem Biophys Res Commun 2016;471:355-360.

29 Sheng Q, Xiao X, Prasadan K, Chen C, Ming Y, Fusco J, Gangopadhyay NN, Ricks D, Gittes GK: Autophagy protects pancreatic beta cell mass and function in the setting of a high-fat and high-glucose diet. Sci Rep 2017;7:16348.

30 Ntsapi C, Loos B: Caloric restriction and the precision-control of autophagy: A strategy for delaying neurodegenerative disease progression. Exp Gerontol 2016;83:97-111.

31 Xu J, Wang P, Wang T, Wang M, Chen S, Yu P, Yu D: Effects of reduced beta2-glycoprotein I on the expression of aortic matrix metalloproteinases and tissue inhibitor matrix metalloproteinases in diabetic mice. BMC Cardiovasc Disord 2014;14:114.

-32 Kong L, Li F, Soleman CE, Li S, Elias RV, Zhou X, Lewis DA, McGinnis JF, Cao W: Bright cyclic light accelerates photoreceptor cell degeneration in tubby mice. Neurobiol Dis 2006;21:468-477.

33 Kong L, Zhou X, Li F, Yodoi J, McGinnis J, and Cao W: Neuroprotective effect of overexpression of thioredoxin on photoreceptor degeneration in Tubby mice. Neurobiol Dis 2010;38:446-455.

-34 Ting DS, Cheung GC, and Wong TY: Diabetic retinopathy: global prevalence, major risk factors, screening practices and public health challenges: a review. Clin Exp Ophthalmol 2016;44:260-277.

-35 Li K, Wang B, Zheng L, Yang K, Li Y, Hu M, He D: Target ROS to induce apoptosis and cell cycle arrest by 5, 7-dimethoxy-1, 4-naphthoquinone derivative. Bioorg Med Chem Lett 2017;28:273-277.

-36 Guo Y, Lin C, Xu P, Wu S, Fu X, Xia W, and Yao M: AGEs Induced Autophagy Impairs Cutaneous Wound Healing via Stimulating Macrophage Polarization to M1 in Diabetes. Sci Rep 2016;6:36416.

-37 Singh BN, Kumar D, Shankar S, Srivastava RK: Rottlerin induces autophagy which leads to apoptotic cell death through inhibition of PI3K/Akt/mTOR pathway in human pancreatic cancer stem cells. Biochem Pharmacol 2012;84:1154-1163.

-38 Yu Y, Li S, Wang Z, He J, Ding Y, Zhang H, Yu W, Shi Y, Cui Z, Wang X, Wang Z, Sun L, Zhang R, Du H, Zhu Z: Interferon regulatory factor-1 activates autophagy to aggravate hepatic ischemia-reperfusion injury via the P38/P62 pathway in mice. Sci Rep 2017;7:43684.

39 Ma K, Huang MY, Guo YX, Hu GQ: Matrine-induced autophagy counteracts cell apoptosis via the ERK signaling pathway in osteosarcoma cells. Oncol Lett 2016;12:1854-1860.

40 Levine B, Yuan J: Autophagy in cell death: an innocent convict? J Clin Invest 2005;115:2679-2688. 